\title{
Arthritis in systemic lupus erythematosus is characterized by local IL-17A and IL-6 expression
}

\author{
Natalie Sippl ${ }^{1}$, Francesca Faustini ${ }^{1}$, Johan Rönnelid ${ }^{2}$, Sara Turcinov ${ }^{1}$, Karine Chemin ${ }^{1}$, Iva \\ Gunnarsson $^{1}$, and V Malmström ${ }^{1}$ \\ ${ }^{1}$ Karolinska Institute \\ ${ }^{2}$ Uppsala University
}

January 12, 2021

\begin{abstract}
Arthritis is a common clinical feature of systemic lupus erythematosus (SLE) and is usually non-erosive as opposed to rheumatoid arthritis (RA). While RA synovial pathology has been extensively studied, little is known about the pathophysiology of lupus arthritis. Here, we aimed to explore the cytokine and cellular compartments in synovial fluids of SLE patients with arthritic manifestations. Acellular synovial fluid and paired serum samples from SLE patients ( $\mathrm{n}=17)$ were analyzed with cytokine bead array for $\mathrm{T}$ helper associated cytokines. From two SLE patients, synovial fluid mononuclear cells (SFMC) were analyzed by multiparameter flow cytometry to dissect T cell, B cell, monocyte and dendritic cell phenotypes. SLE-derived SFMC were further stimulated in vitro to measure their capacity for producing IFN? and IL-17A. All patients fulfilled the ACR 1982 classification criteria for SLE. Clinical records were reviewed to exclude the presence of comorbidities such as osteoarthritis or overlap with RA. IL-17A and IL-6 levels were high in SLE synovial fluid. A clear subset of the synovial CD4+ T cells expressed CCR6+, a marker associated with Th17 cells. IL-17-production was validated amongst CD4+CCR6+ T cells following in vitro stimulation. Furthermore, a strong IFN? production was observed in both CD4+ and CD8+ cells. Our study shows high IL-17A and IL-6 levels in synovial fluids of patients with lupus arthritis. The Th17 pathway have been implicated in several aspects of SLE disease pathogenesis and our data points to Th17 involvement also for lupus arthritis.
\end{abstract}

\section{TITLE PAGE}

Arthritis in systemic lupus erythematosus is characterized by local IL-17A and IL-6 expression Natalie Sippl ${ }^{1 *}$, Francesca Faustini ${ }^{*}{ }^{*}$, Johan Rönnelid ${ }^{2}$, Sara Turcinov ${ }^{1}$, Karine Chemin ${ }^{1}$, Iva Gunnarsson ${ }^{1}$, Vivianne Malmström ${ }^{1}$

\section{Affiliations}

${ }^{1}$ Division of Rheumatology, Department of Medicine, Center of Molecular Medicine, Karolinska University Hospital Solna, Karolinska Institute, Stockholm, Sweden

2 Department Immunology, Genetics and Pathology, Uppsala University, Uppsala, Sweden.

* Natalie Sippl and Francesca Faustini contributed equally to this work

Short Title: Synovial IL-17A and IL6 expression in lupus arthritis patients

Correspondence to: Vivianne Malmström, Center for Molecular Medicine, L8:04, 17176 Stockholm, Sweden. Email: vivianne.malmstrom@ki.se

Keywords: 
Systemic lupus erythematosus, arthritis, synovial fluid, cytokines, T cells

\section{List of abbreviations}

SLE: systemic lupus erythematosus; LE: lupus erythematosus; RA: rheumatoid arthritis ; SpA: spondyloarthritis; OA: osteoarthritis; ARA/ACR: American Rheumatism Association/ American College of Rheumatology; ASAS: assessment of spondyloarthritis international society; SF: synovial fluid; PB: peripheral blood; SFMC/PBMC: synovial fluid-/peripheral blood- mononuclear cells; ACPA: anti citrullinated peptides antibodies; RF: rheumatoid factor; ANA: antinuclear antibodies; CRP: C-reactive protein; CBA: cytokine bead array; tSNE: t-distributed stochastic neighbor embedding; DMSO/FCS: dimethyl-sulfoxide/fetal calf serum; Th: T helper cell; Th17: T-helper 17; $\mathrm{T}_{\mathrm{PH}}$ : T-peripheral helper; Treg: T-regulatory cell; DC: dendritic cells; cDC: classical dendritic cell; CD4/CD8/CD3/CD28/CD11c: CD- cluster of differentiation; CCR4/CCR6: CCR- chemokine receptor; CXCR/CXCR3/CXCR5: CXCR:C-X-C chemokine receptor; Eomes: Eomesodermin; HLA-DR: Human Leucocyte Antigen-DR isotype; FoxP3:forkhead box P3; PD-1: programmed cell death protein-1; GZMA: granzyme A; TNF: tumor necrosis factor; IL-13/IL-2/IL-4/IL-6/IL-8/IL-10/IL-17A, IL: interleukin; IFN $\gamma$ : interferon gamma; IgA/IgM: Immunoglobulin, isotype A/isotype M , IQR: interquartile range

\section{Summary}

Arthritis is a common clinical feature of systemic lupus erythematosus (SLE) and is usually non-erosive as opposed to rheumatoid arthritis (RA). While RA synovial pathology has been extensively studied, little is known about the pathophysiology of lupus arthritis. Here, we aimed to explore the cytokine and cellular compartments in synovial fluids of SLE patients with arthritic manifestations.

Acellular synovial fluid and paired serum samples from SLE patients $(n=17)$ were analyzed with cytokine bead array for $\mathrm{T}$ helper associated cytokines. From two SLE patients, synovial fluid mononuclear cells (SFMC) were analyzed by multiparameter flow cytometry to dissect T cell, B cell, monocyte and dendritic cell phenotypes. SLE-derived SFMC were further stimulated in vitro to measure their capacity for producing IFN $\gamma$ and IL-17A. All patients fulfilled the ACR 1982 classification criteria for SLE. Clinical records were reviewed to exclude the presence of comorbidities such as osteoarthritis or overlap with RA.

IL-17A and IL-6 levels were high in SLE synovial fluid. A clear subset of the synovial CD4+ T cells expressed CCR6+, a marker associated with Th17 cells. IL-17-production was validated amongst CD4+CCR6+ T

cells following in vitro stimulation. Furthermore, a strong IFN $\gamma$ production was observed in both CD4+ and CD8+ cells.

Our study shows high IL-17A and IL-6 levels in synovial fluids of patients with lupus arthritis. The Th17 pathway have been implicated in several aspects of SLE disease pathogenesis and

our data points to Th17 involvement also for lupus arthritis.

\section{MAIN MANUSCRIPT}

\section{Introduction}

Arthritis is a common manifestation in systemic lupus erythematosus (SLE), observed in up to $90 \%$ of patients ${ }^{1}$. It often occurs at disease onset and is part of classification criteria ${ }^{2,3}$, but it is usually nonerosive, although advanced imaging has revealed that chronic synovitis ${ }^{4}$ and erosions ${ }^{5}$ are more common than previously estimated.

Despite being frequent and causing a significant disease burden by influencing quality of life ${ }^{6}$, arthritis has received less attention than other SLE manifestations, such as nephritis, that directly impact morbidity and survival. While several studies have investigated possible associations of arthritis with genetic risk factors, immunological features, and autoantibodies ${ }^{6}$, little is known of the local inflammatory cell phenotypes and cytokines. 
In the 70ties and 90ties, descriptive studies of SLE synovial biopsies have demonstrated microvascular changes, moderate proliferation of the synovial lining layer as well as mononuclear cell infiltrates and fibrin deposits $^{7}$ in affected tissue. The synovial fluid was found to typically contain less than 2000 leucocytes per microliter, and to be characterized by lymphocytes and so called lupus erythematosus (LE) cells, i.e. the presence of nucleic acids in the cytoplasm of neutrophils and macrophages ${ }^{8}$. Presence of anti-nuclear antibodies (ANA) were also reported in synovial fluid of SLE patients ${ }^{9}$. In 2007 , Toukap $\mathrm{N}$ et al. ${ }^{10}$ analyzed the gene expression of synovial biopsies and found that SLE patients display a distinct molecular signature, with up-regulation of interferon inducible genes and down-regulation of genes involved in extracellular matrix homeostasis. However, the involvement of specific cell types or cytokines in lupus arthritis still remains unknown.

In order to better understand SLE joint pathology, we analyzed cytokines associated to T-cell responses and the cellular composition (when present) of synovial fluid (SF) samples from SLE patients as compared to paired peripheral blood samples and found an upregulation of IL-6 and IL-17A in synovial fluid of SLE patients, as well as potential pathogenic $\mathrm{T}$ cell subsets.

\section{Materials and Methods}

Patient material

SF was obtained from routine large joint aspiration from SLE, SpA and RA patients fulfilling the 1982 $\mathrm{ARA} / \mathrm{ACR}^{2}, \mathrm{ASAS}^{11}$ and ACR classification criteria ${ }^{12}$ respectively. Informed consent was given prior to SF and blood sampling, as approved by the local ethics committee.

Once collected, SF was centrifuged and stored as acellular at $-80^{\circ} \mathrm{C}$. In parallel, paired serum samples were also processed. The mononuclear cell fractions from SF (when present) and peripheral blood were collected following gradient density centrifugation over Ficoll-Hypaque and were cryopreserved in DMSO/FCS in $\mathrm{N}_{2}(\mathrm{l})$ until use.

SLE synovial fluid samples, once retrieved, were selected for inclusion in the present study after revision of the electronic clinical charts, in order to exclude biological material from patients with evidence of joint involvement not primarily dependent on SLE. We therefore revised clinical information to exclude primary osteoarthritis, overlap with rheumatoid arthritis, crystal related arthritis, and other comorbidities which could entail potential confounders.

From an initial list of 27 patients, we therefore selected 17 SLE patients with joint involvement, excluding 10 patients (1 with SLE RA overlap, 1 with onset of arthritis after the occurrence of a haematological malignancy, 8 for evidence of primary OA or crystal related arthritis). The clinical characteristics are listed in Table 1 (SLE patients used for the cytokine analysis) and Supplementary Tables S1 (RA patients used for the cytokine analysis) and S2 (patient samples for cell analysis).

Autoantibodies

IgA and IgM rheumatoid factor (RF) (Elia, Thermo Fisher Scientific, Uppsala, Sweden) and anti-citrullinated peptide antibodies (ACPA; CCPlus, EuroDiagnostica, Malmö, Sweden) were analyzed in sera and SF from SLE patients according to the manufacturers' instructions.

\section{Cytokine Bead Array}

SF samples $(n=17)$, matched serum samples $(n=15)$ from SLE patients (Table 1), and SF samples from RA patients ( $\mathrm{n}=10$, Supplementary Table S1) matched for storage time, were analyzed with cytokine bead array (CBA) for T-helper-associated cytokines (BD Biosciences, San Jose, USA) following the manufacturer's instructions. In brief, thawed serum samples were diluted 1:2 with assay diluent and run-in duplicates in a 96-well plate. Cytokines tested were IL-17A, IFN $\gamma$, IL-10, IL-6, IL-4, IL-2 and TNF. The assay was run on a FACS Verse flow cytometer and analyzed using the CBA software (BD Biosciences, San Jose, USA). Limits of detections are displayed in Supplementary Fig. S2C. 
Lymphocyte isolation and cell assays

SF mononuclear cells (SFMC) and paired peripheral blood samples (PBMC) from two SLE, three SpA and two seronegative RA patients (matched for age and sex, Supplementary Table S2), were analyzed by flow cytometry panels for $\mathrm{T}$ cells and when possible for B cells, monocytes and dendritic cells (Supplementary Table S4). Cryopreserved PBMC and SFMC were thawed and stained with the corresponding antibody mixtures and viability dye (Supplementary Table S4 and S5). For functional studies, duplicates of $0.5 \times 10^{6}$ SFMC were stimulated with anti-CD3/CD28 beads (1bead/cell, Dynabeads, Thermo-Fisher, Waltham, USA) for $16 \mathrm{~h}$ in 96 -well flat-bottom plate. The last $4 \mathrm{~h}$ brefeldin-A $(5 \mathrm{ug} / \mathrm{ml}$, Sigma Aldrich, St. Louis, USA) was added. Analyses were run on a Fortessa LSR cell analyzer (BD Biosciences, San Jose, USA) and data were processed by FlowJo software (BD Biosciences, San Jose, USA). Gating strategies are displayed in Supplementary Fig. S1.

Statistical analysis

Continuous variables are expressed as median and interquartile range (IQR), categorical variables as numbers and percentages. Non-parametric statistics was used to explore differences and correlations as appropriate. P-values $<0.05$ were deemed statistically significant. Prism7 software (GraphPad, San Diego, USA) was used.

\section{Results}

Clinical characteristics

Clinical characteristics of the 17 SLE patients at the time of SF sampling are summarized in Table 1. The patients were fairly representative of a typical SLE population. Samples were collected at a time of long disease history including long standing arthritis, often polyarticular and which had occurred among the first disease manifestations. Six of the patients (age at onset 28y, IQR 17-38) had developed secondary knee osteoarthritis over time.

IL-17A and IL-6 are highly expressed in SLE SF

First, we wanted to investigate T-cell associated cytokines in SF of SLE patients. Thus, SF from SLE patients $(n=17)$ were tested for cytokine content in comparison to paired serum samples $(n=14)$ and SF from RA patients $(\mathrm{n}=10)$, matched for storage time (Supplementary Table S2). In SLE, IL-17A was detected in SF $(44.9 \mathrm{pg} / \mathrm{ml}(10.7-405.2))$ and matched serum $(37.5 \mathrm{pg} / \mathrm{ml}(22.4-94.2))$ (Fig. 1A), although no statistically significant difference was shown $(p=0.4)$.

IL-6 was significantly higher in SLE-SF $(771 \mathrm{pg} / \mathrm{ml}$ (108.5-2135)) as compared to corresponding serum samples $(1.7 \mathrm{pg} / \mathrm{ml}(1.2-4.9), p=0.0006)$. Low concentrations of IL-10 could be detected in $41 \%$ of SLE SF samples $(0 \mathrm{pg} / \mathrm{ml}(0-3.4))$ and was higher in serum $(0.29 \mathrm{pg} / \mathrm{ml}(0.1-0.4))$. Overall, the RA SF samples displayed similar ranges of cytokines as SLE SF, with higher median values of IL-6 (3627 pg/ml (334.6-5000), $\mathrm{p}=0.11)$ and IL-10 $(1.9 \mathrm{pg} / \mathrm{ml}(0-7.9), \mathrm{p}=0.19)$ and less IL-17A, $(0 \mathrm{pg} / \mathrm{ml}(0-172), \mathrm{p}=0.11)$ however without statistical significance (Fig. 1A). The cytokine levels did not differ between SLE patients with and without secondary osteoarthritis (data not shown).

Next, we explored the relationship of cytokine levels, in and between compartments (Fig. 1B-C). In SLE SF, the levels of IL-6 correlated with those of IL-10 ( $\mathrm{r}=0.84, p=<0.0001, \mathrm{CI}=0.69-0.92)$ and, weakly, with IL17A $(\mathrm{r}=0.39, p=0.03, \mathrm{CI}=0.02-0.66)$ (Fig. 1B). Also, IL-10 and IL-17A correlated weakly $(\mathrm{r}=0.43, p=0.01$ , CI=0.07-0.69). When comparing serum and SF, levels of IL-6 correlated $(r=0.7, p=0.0001)$ but neither IL-17A nor IL-10 (Fig. 1C).

Cytokine analyses included also IFN- $\gamma$, TNF, IL-2 and IL-4. In sera, IL-4 and IL-2 were higher compared to $\mathrm{SF}$, however their concentrations were consistently low (Supplementary Fig. 1A). Very low levels of IFN- $\gamma$ could be detected in SF of four SLE patients. TNF levels were below the detection limit in all samples (Supplementary Fig. 2B). 
Subsequently, we investigated the presence of IgA and IgM RF in serum and SF. These did not correlate with the cytokine levels nor with other autoantibodies (data not shown). Interestingly, the concentration of $\mathrm{RF}$, when present, was mostly higher in serum compared to SF (Fig. 1D).

For one SLE patient, SF samples from six timepoints were available, the first obtained approximately 15 years after disease onset. This patient presented with a recurrent polyarthritis, but no evidence of secondary OA could be tracked in the clinical records. High IL-6 levels were repeatedly measured over a 10 years period, while IL-17A levels fluctuated and IL-10 concentrations were low (Fig. 2A).

Cellular composition of SLE synovial fluid

Subsequently, we were interested in the cellular composition found in SF of SLE patients. SFMC were available from two SLE patients, one with as multiple timepoints as described above and another with cells from two timepoints. These samples were compared with SFMC from three SpA and two age and sex matched seronegative RA patients. All samples were investigated for T-cell phenotypes and when possible also for B cell, monocyte and dendritic cell subsets (Supplementary Table S4).

In SLE SF, lymphocytes consisted mainly of CD3+ T cells, with CD8+ cells being more common in SF than in PB (Fig. 2B, Supplementary Fig. 3A). B cells which were rare $(<1 \%)$ displayed a memory phenotype, for one patient including age associated CD11c+ CD27+ B cells (Fig. 2B).

Examining the monocyte and dendritic cell (DC) subsets in SLE-SF, 64-67\% of the HLA-DR+ cells expressed CD14 without expressing CD16, a sign for classical monocytes (Fig.2B, Supplementary Fig. S3C). Nonmonocyte cells represented $\sim 30 \%$ of HLA-DR + cells in SF, and were mostly classical DC (CD11c+, cDC), in patient one mainly $\mathrm{cDC} 1$ while the other exhibited equal proportions of $\mathrm{cDC} 1$ and $\mathrm{cDC} 2$. The frequency of plasmacytoid DC varied between patients from 7-26\% (Fig. 2B, Supplementary Fig. S3C).

$\mathrm{T}$ helper17 signatures in SF of SLE patients

Focusing on T-cell phenotypes, we visualized the distribution by tSNE analysis of the CD3+ T cells. As depicted in Fig. 2C, PBMC and SFMC T-cell cluster in different directions. Within the CD4+ compartment, we observed higher frequencies of CCR4+, CXCR5+, CCR6+ and similar levels of CXCR3+ in SF compared to PB. The most prominent T-helper subset in SF was CCR6+ cells, a marker for Th17 cells. 35-55\% of CD4+ T cells expressed CCR6 (Fig. 2C, Supplementary Fig. 3D), with $~ 40 \%$ co-expressing CCR4. Moreover, Tregs (CD25+ FoxP3+), Eomes+, Granzyme A+ (GZMA), were also enriched in SF as compared to PB (Fig. 2C). PD1+HLA-DR+ cells were also abundant, and we identified a distinct population of T-peripheral helper $\left(\mathrm{T}_{\mathrm{PH}}\right.$; PD1 ${ }^{\text {high }} \mathrm{HLA}-\mathrm{DR}{ }^{\text {high }}$ ) cells in SF, of which ${ }^{\sim} 30 \%$ co-expressed CCR6 (Fig. 3). In SF, ${ }^{70-80 \%}$ of CD8+ T cells expressed Eomes and GZMA and a high proportion of CD8+ PD-1+ HLA-DR+/- cells were detected in all SF samples (Fig. 2C, Supplementary Fig. S3D). CX3CR1 was not expressed on CD4+ nor CD8+ T cells in SF. SF T-cell phenotypes in SLE, RA and SpA, presented similar patterns.

From one SLE patient, SFMC from two timepoints were available (Supplementary Fig.S3E). For most CD8+ and CD4+ subsets, similar frequencies were detected with the exception of GZMA+ Eomes- T cells which were more prominent at the first timepoint. In line with the other SLE patient, CCR6+ CD4+ T cells was one of the most prominent subsets in SF.

Since we observed both IL-17A and increased CCR6+ CD4+ T cells in SF of SLE patients, we investigated the capacity of SF-derived T cells to produce IL-17A. Following in vitro stimulation of SFMCs from one SLE and one SpA sample, intracellular IL-17A and IFN $\gamma$ production was analyzed. In both samples, IL-17A production was induced and confined to the CCR6+ CD4+ cells (Fig.4, Supplementary Fig.S4). We also observed abundant IFN $\gamma$ production in IL-17-negative CD4 $+\mathrm{T}$ cells. Of note CD8 $+\mathrm{T}$ cells also produced IFN $\gamma$, but not IL-17A.

\section{Discussion}

We found that local joint inflammation in SLE is dominated by IL-6 and IL-17. IL-6, a pleiotropic cytokine, can influence the function of several immune cells such as B and $\mathrm{T}$ cells, orientating their fate towards 
Th17 and $\mathrm{T}$ follicular helper phenotypes ${ }^{13}$. Its local expression in SLE inflamed large joints might also suggest a more acute joint pathology and an effect on recruiting neutrophils. Indeed, most of the SLE SF samples contained only neutrophils and became acellular following the cell purification step. Previous studies investigating IL-6 plasma concentration in relation to SLE arthritis have shown that elevated IL-6 was associated with active ongoing arthritis but not with CRP levels ${ }^{14,15}$ and also a correlation between IL-6 plasma level and joint involvement ${ }^{15}$. In our study, IL-6 SF levels also correlated with IL-6 serum levels, albeit IL-6 concentration was much lower in serum, suggesting a more relevant local role for this cytokine.

IL-17A, known to trigger the recruitment of neutrophils and monocytes to the site of inflammation ${ }^{16}$ was abundant in SLE SF samples, similarly to what has been observed in ankylosing spondylitis ${ }^{17}$. In recent years, several studies have highlighted the possible implications of the IL-17 axis in SLE pathogenesis ${ }^{18}$. IL-17A has been found to be increased in sera of SLE patients compared to healthy controls, expressed in kidney tissue of patients with lupus nephritis and to predict an unfavorable histopathological response to immunosuppressive treatment ${ }^{19}$. Current clinical trials are evaluating IL-17A inhibition in refractory lupus nephritis (https://www.clinicaltrials.gov/ct2/show/NCT04181762).

This is the first study that, to our knowledge, investigates the cellular composition of SF in SLE. In most cases, the numbers of SF mononuclear cells were low, thereby only the fluid could be preserved. Still, we were able to analyze mononuclear cells from two synovial fluids from SLE patients. Similar to other inflammatory arthritides ${ }^{20,21}$ an increase of Tregs could be observed in SLE SF. We could also identify a PD-1 ${ }^{\text {high }}$ HLA$\mathrm{DR}^{\text {high }} \mathrm{T}_{\mathrm{PH}}$ population in SF of SLE patients. This T-cell subset has been described in ACPA+ RA patients and functions by promoting B-cell responses and antibody production ${ }^{22}$. Similarly, PD ${ }^{\text {high }}$ CXCR5- ${ }_{\mathrm{PH}}$ have been described in SLE where they appear to drive the dynamics of pathologic B-cell responses, correlate with disease activity and are expressed in kidney tissue in lupus nephritis ${ }^{23}$.

In our study, CCR6+ CD4+ T cell subsets were abundant in SLE-SF. CCR6+ is a chemokine receptor expressed on Th17 cells and Th17 differentiation is dependent on the cytokines IL-6, IL-1 $\beta$ and TGF $\beta^{24}$. Th17 cells have previously been reported to increase during SLE flare and to be reduced after successful treatment ${ }^{25}$. Leipe $\mathrm{J}$ et al. ${ }^{26}$ reported an increase of Th17 cells in psoriatic arthritis and RA patients in peripheral blood and synovium, with an enrichment of Th17 in SF in the affected joint. Similarly, CCR6+ cells were increased in SF of the two analyzed SLE patients compared to peripheral blood.

Due to the increase of IL-17A and CCR6+ T cells in SF of SLE patients, we stimulated SFMC with antiCD3/CD28 beads to study their capacity to produce cytokines. Thereby, we identified IL-17A producing $\mathrm{T}$ cells, primarily represented by CCR $6+\mathrm{CD} 4+\mathrm{T}$ cells. It is however possible that other cells also could contribute to IL-17A production as both CD4 and CD8, as well as $\gamma \delta \mathrm{T}$ cells, innate lymphoid cells and mast cells have been identified as IL-17A producers in psoriasis and psoriatic arthritis ${ }^{27}$.

While CCR6+ was one of the most prominent CD4+ T-cell subsets in SLE-SF, the vast majority of SF CD8+ $\mathrm{T}$ cell expressed the transcription factor Eomes and GZMA similar to observations in RA ${ }^{28}$. GZMA induces pro-inflammatory cytokines such as IL-6, IL-8, IL-1 $\beta$. Altogether, our analysis suggests an involvement of T cells in the pathogenesis of lupus arthritis.

The main limitation of our study is that it was conducted retrospectively on historical material. Systematic assessment of joint involvement and SLE disease activity were not performed at the time of sampling, thus clinical associations could not be further explored. Moreover, the sampling was performed only on large joints, which are technically more accessible but imply some limitations, since SLE arthritis more typically involves small joints. The long disease duration in the SLE patients with ongoing and previous treatments could also modify the SF composition. Being most often acellular or paucicellular, a strength of our study is the inclusion of two cellular samples of SLE SF.

From a clinical perspective, lupus arthritis is common already from the time of diagnosis but is a manifestation often responding to treatment. This may be a reason why arthritis has been given relatively less attention from a research perspective. Our study cohort is admittedly small but still represents one of the most comprehensive collections of SF material in SLE. It is tempting to speculate that lupus arthritis is less 
autoimmune in character as compared to rheumatoid arthritis and our data lend support to such a theory by suggesting that it is part of a systemic immune deviation towards Th17 and not joint specific. Indeed, Th17 responses implicate the involvement of innate immunity through neutrophils, cells that historically have been linked to SLE pathophysiology as LE cells ${ }^{8}$.

In conclusion, our study provided new exploratory insights into the immunology of lupus arthritis. IL-17A and IL-6 levels were high in synovial fluid of SLE patients. The presence of CCR6+ CD4+ T cells, GZMA+ EOMES + as well as PD1+ HLA-DR + CD4+ and CD8+ T cells suggest a pathogenic role of T cells in lupus arthritis. Our findings suggest that SLE patients with arthritis may improve if treated with IL-17A blockade for other clinical manifestations.

\section{References:}

1. Ball EM, Bell AL. Lupus arthritis-do we have a clinically useful classification? Rheumatology (Oxford) 2012; 51:771-9.

2. Tan EM, Cohen AS, Fries JF, Masi AT, McShane DJ, Rothfield NF, et al. The 1982 revised criteria for the classification of systemic lupus erythematosus. Arthritis Rheum 1982; 25:1271-7.

3. Petri M, Orbai AM, Alarcon GS, Gordon C, Merrill JT, Fortin PR, et al. Derivation and validation of the Systemic Lupus International Collaborating Clinics classification criteria for systemic lupus erythematosus. Arthritis Rheum 2012; 64:2677-86.

4. Zayat AS, Mahmoud K, Md Yusof MY, Mukherjee S, D'Agostino MA, Hensor EMA, et al. Defining inflammatory musculoskeletal manifestations in systemic lupus erythematosus. Rheumatology (Oxford) $2019 ; 58: 304-12$.

5. Tani C, D'Aniello D, Possemato N, Delle Sedie A, Caramella D, Bombardieri S, et al. MRI pattern of arthritis in systemic lupus erythematosus: a comparative study with rheumatoid arthritis and healthy subjects. Skeletal Radiol 2015; 44:261-6.

6. Ceccarelli F, Perricone C, Cipriano E, Massaro L, Natalucci F, Capalbo G, et al. Joint involvement in systemic lupus erythematosus: From pathogenesis to clinical assessment. Semin Arthritis Rheum 2017; 47:53-64.

7. Goldenberg DL, Cohen AS. Synovial membrane histopathology in the differential diagnosis of rheumatoid arthritis, gout, pseudogout, systemic lupus erythematosus, infectious arthritis and degenerative joint disease. Medicine (Baltimore) 1978; 57:239-52.

8. Schumacher HR, Jr., Howe HS. Synovial fluid cells in systemic lupus erythematosus: light and electron microscopic studies. Lupus 1995; 4:353-64.

9. Labowitz R, Schumacher HR, Jr. Articular manifestations of systemic lupus erythematosus. Ann Intern Med 1971; 74:911-21.

10. Nzeusseu Toukap A, Galant C, Theate I, Maudoux AL, Lories RJ, Houssiau FA, et al. Identification of distinct gene expression profiles in the synovium of patients with systemic lupus erythematosus. Arthritis Rheum 2007; 56:1579-88.

11. Rudwaleit M, van der Heijde D, Landewe R, Akkoc N, Brandt J, Chou CT, et al. The Assessment of SpondyloArthritis International Society classification criteria for peripheral spondyloarthritis and for spondyloarthritis in general. Ann Rheum Dis 2011; 70:25-31.

12. Aletaha D, Neogi T, Silman AJ, Funovits J, Felson DT, Bingham CO, 3rd, et al. 2010 Rheumatoid arthritis classification criteria: an American College of Rheumatology/European League Against Rheumatism collaborative initiative. Arthritis Rheum 2010; 62:2569-81.

13. Schinnerling K, Aguillon JC, Catalan D, Soto L. The role of interleukin-6 signalling and its therapeutic blockage in skewing the T cell balance in rheumatoid arthritis. Clin Exp Immunol 2017; 189:12-20. 
14. Eilertsen GO, Nikolaisen C, Becker-Merok A, Nossent JC. Interleukin-6 promotes arthritis and joint deformation in patients with systemic lupus erythematosus. Lupus 2011; 20:607-13.

15. Ball EM, Gibson DS, Bell AL, Rooney MR. Plasma IL-6 levels correlate with clinical and ultrasound measures of arthritis in patients with systemic lupus erythematosus. Lupus 2014; 23:46-56.

16. Pelletier M, Maggi L, Micheletti A, Lazzeri E, Tamassia N, Costantini C, et al. Evidence for a cross-talk between human neutrophils and Th17 cells. Blood 2010; 115:335-43.

17. Bowness P, Ridley A, Shaw J, Chan AT, Wong-Baeza I, Fleming M, et al. Th17 cells expressing KIR3DL2+ and responsive to HLA-B27 homodimers are increased in ankylosing spondylitis. J Immunol 2011; 186:2672-80.

18. Robert M, Miossec P. Interleukin-17 and lupus: enough to be a target? For which patients? Lupus 2020; 29:6-14.

19. Zickert A, Amoudruz P, Sundstrom Y, Ronnelid J, Malmstrom V, Gunnarsson I. IL-17 and IL-23 in lupus nephritis - association to histopathology and response to treatment. BMC Immunol 2015; 16:7.

20. Cao D, van Vollenhoven R, Klareskog L, Trollmo C, Malmstrom V. CD25brightCD4+ regulatory T cells are enriched in inflamed joints of patients with chronic rheumatic disease. Arthritis Res Ther 2004; 6:R335-46.

21. Bommarito D, Hall C, Taams LS, Corrigall VM. Inflammatory cytokines compromise programmed cell death-1 (PD-1)-mediated T cell suppression in inflammatory arthritis through up-regulation of soluble PD-1. Clin Exp Immunol 2017; 188:455-66.

22. Rao DA, Gurish MF, Marshall JL, Slowikowski K, Fonseka CY, Liu Y, et al. Pathologically expanded peripheral T helper cell subset drives B cells in rheumatoid arthritis. Nature 2017; 542:110-4.

23. Bocharnikov AV, Keegan J, Wacleche VS, Cao Y, Fonseka CY, Wang G, et al. PD-1hiCXCR5- T peripheral helper cells promote B cell responses in lupus via MAF and IL-21. JCI Insight 2019; 4.

24. Burkett PR, Meyer zu Horste G, Kuchroo VK. Pouring fuel on the fire: Th17 cells, the environment, and autoimmunity. J Clin Invest 2015; 125:2211-9.

25. Yang J, Chu Y, Yang X, Gao D, Zhu L, Yang X, et al. Th17 and natural Treg cell population dynamics in systemic lupus erythematosus. Arthritis Rheum 2009; 60:1472-83.

26. Leipe J, Grunke M, Dechant C, Reindl C, Kerzendorf U, Schulze-Koops H, et al. Role of Th17 cells in human autoimmune arthritis. Arthritis Rheum 2010; 62:2876-85.

27. Menon B, Gullick NJ, Walter GJ, Rajasekhar M, Garrood T, Evans HG, et al. Interleukin-17+CD8+ $\mathrm{T}$ cells are enriched in the joints of patients with psoriatic arthritis and correlate with disease activity and joint damage progression. Arthritis Rheumatol 2014; 66:1272-81.

28. Chemin K, Ramskold D, Diaz-Gallo LM, Herrath J, Houtman M, Tandre K, et al. EOMES-positive CD4(+) T cells are increased in PTPN22 (1858T) risk allele carriers. Eur J Immunol 2018; 48:655-69.

TABLE 1: Characteristics of the Systemic Lupus Erythematosus (SLE) patients analyzed with the cytokine bead array

\begin{tabular}{ll}
\hline Clinical characteristic & $\mathbf{N = 1 7}$ \\
\hline Gender (Females N (\%)) & $16(94.1)$ \\
Age at disease onset (years, M, (IQR) & $31(23-42.5)$ \\
Age at SF sampling (years, M, (IQR) & $57(48.5-63.2)$ \\
Disease duration (years, M, (IQR) & $16(6-34)$ \\
Active smoking at sampling (N (\%)) & $6(35.3)$
\end{tabular}




\begin{tabular}{ll}
\hline Clinical characteristic & $\mathbf{N = 1 7}$ \\
\hline Organ involvement & \\
Lupus Nephritis (N (\%)) & $3(17.6)$ \\
Cutaneous manifestations (N (\%)) & $6(35.3)$ \\
Neurolupus (N (\%)) & $3(17.6)$ \\
Serositis (N (\%)) & $2(11.8)$ \\
Secondary APS (N (\%)) & $3(17.6)$ \\
Treatment at sampling & \\
Glucocorticoids (N (\%)) & $10(58.8)$ \\
Antimalarial (N (\%)) & $10(58.8)$ \\
Methotrexate (N (\%)) & $3(17.6)$ \\
Combined therapy\# (N (\%)) & $6(35.3)$ \\
No active treatment (N (\%)) & $2(11.8)$ \\
Autoantibodies & \\
ANA (N (\%)) & $17(100)$ \\
anti-dsDNA (N (\%)) & $6(35.3)$ \\
RF (N (\%)) & $2(11.8)$ \\
anti-SSA/SSB (N (\%)) & $4(23.5)$ \\
anti-RNP (N (\%)) & $3(17.6)$ \\
anti-PL (N (\%)) & $9(52.9)$ \\
\hline
\end{tabular}

\section{Table legend}

*manifested during the course of the disease; ^dose range 2.5-15 mg daily,

\# combination of either GC and antimalarial, GC and MTX, GC-MTX and antimalarial

Abbreviations M: median, IQR: interquartile range, GC: glucocorticoids, MTX: methotrexate; ANA: antinuclear antibodies; Anti-dsDNA: anti-double stranded DNA; RF: rheumatoid factor; Anti-SSA/SSB: antiSjögren's syndrome-related antibody A, B; Anti-RNP: anti-ribonucleoprotein; aPL: antiphospholipid antibodies, ^mostly anti-cardiolipin;

\section{FIGURES}


A

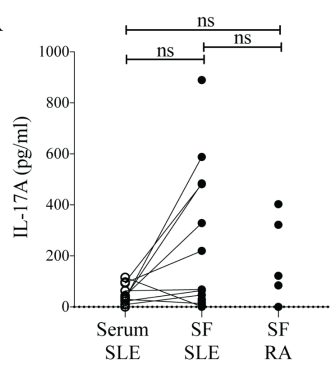

B
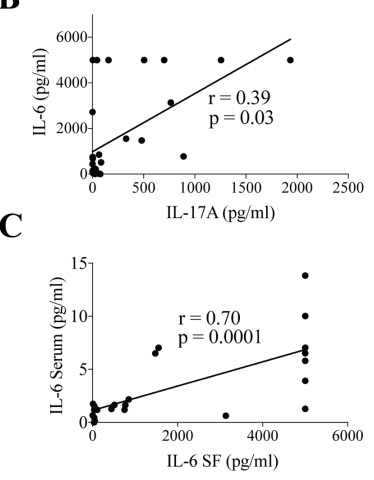
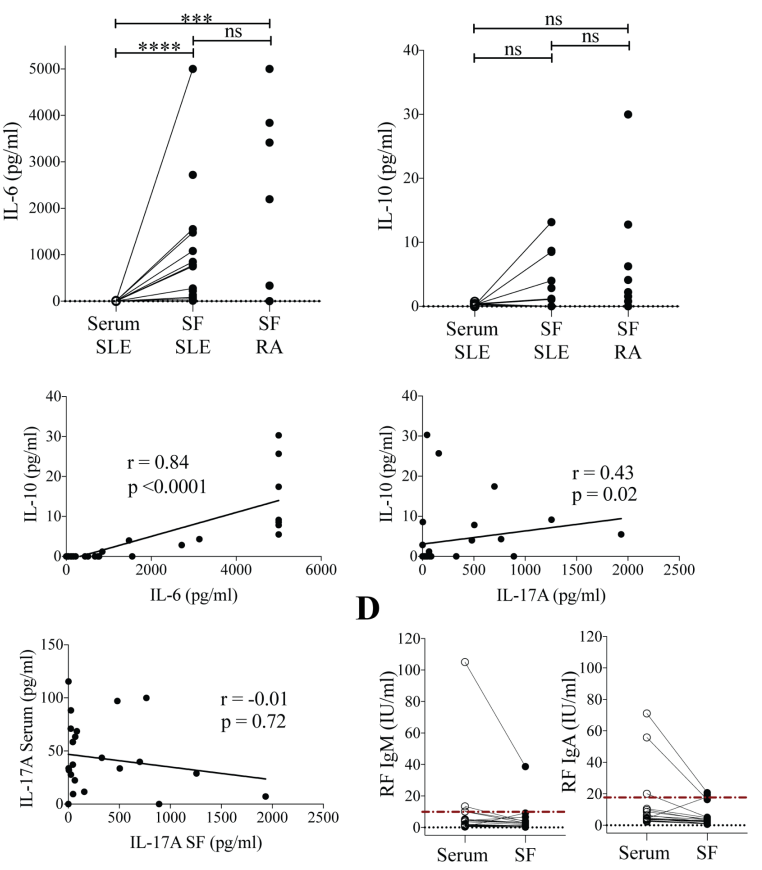

\section{Figure 1:}

Cytokine analysis of SLE SF samples (A) IL-17A, IL-6 and IL-10 cytokine levels were measured in SLE SF samples $(n=17)$, matched serum samples $(n=14$, combined with lines) and SF RA samples $(n=10)$ and significant difference was analyzed using Mann Whitney test. Bars indicate median. (B) Spearman Correlations between IL17A, IL-6 and IL-10 levels in SF are displayed. (C) Spearman Correlation between IL-17A and IL-6 levels in serum vs SF. (D) IgA and IgM RF levels in serum and SF are shown. Red line indicates cut off: $\operatorname{IgA} R F=18 \mathrm{IU} / \mathrm{ml} ; \mathrm{IgM} \mathrm{RF}=10 \mathrm{IU} / \mathrm{ml})$

A

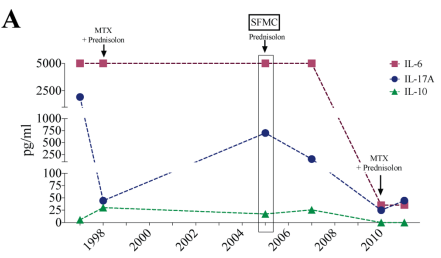

B

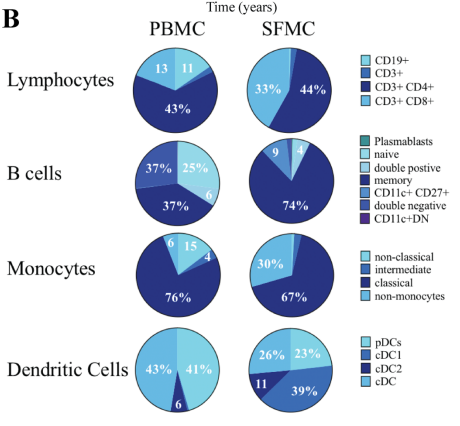

$\mathbf{C}$
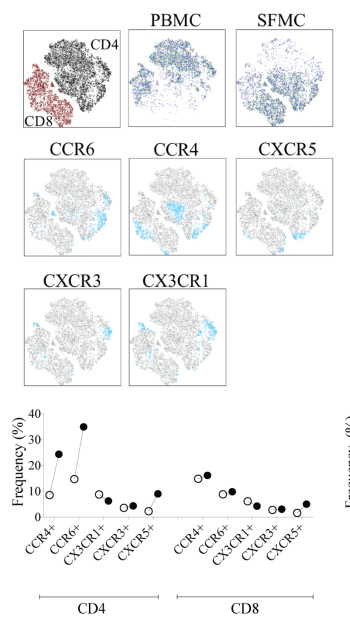
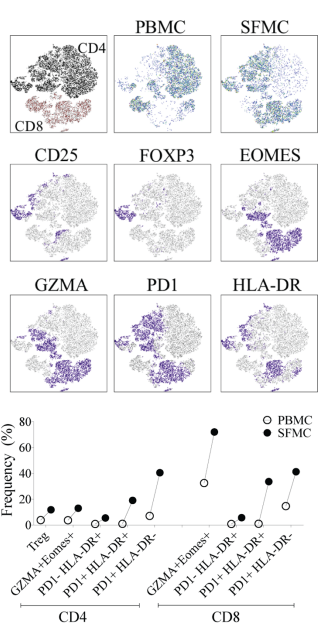

Figure 2:

Longitudinal Cytokine analysis and Cellular composition in SLE SF (A) Longitudinal cytokine analysis of SLE-SF. Change of medication and the timepoint obtaining SFMC are indicated. (B) Proportions 
of cell subsets in SF and matched PB. B-cell subsets: plasmablasts (CD27++CD38++); naïve (IgD+CD27), double positive $(\operatorname{IgD}+\mathrm{CD} 27+)$, memory (IgD-CD27+), double negative (DN, IgD-CD27-). Monocyte subsets: non-classical (CD14-C16+), intermediate (CD14+C16-+), classical (CD14+C16-) and nonmonocytes (CD14-CD16-). Dendritic Cell subsets: classical DC (cDC, CD11c+), cDC1 (CD11c+CD1c+), cDC2 (CD11c+CD141+) and pDC (CD11c-CD123+CD303+). Frequencies $<4 \%$ are not displayed. (C) tSNE clustering of CD3+ cells from the two T-cell panels (Supplementary Table S4) and CD4+ and CD8 T-cell subsets in paired $\mathrm{PB}$ and $\mathrm{SF}(\mathrm{n}=1)$
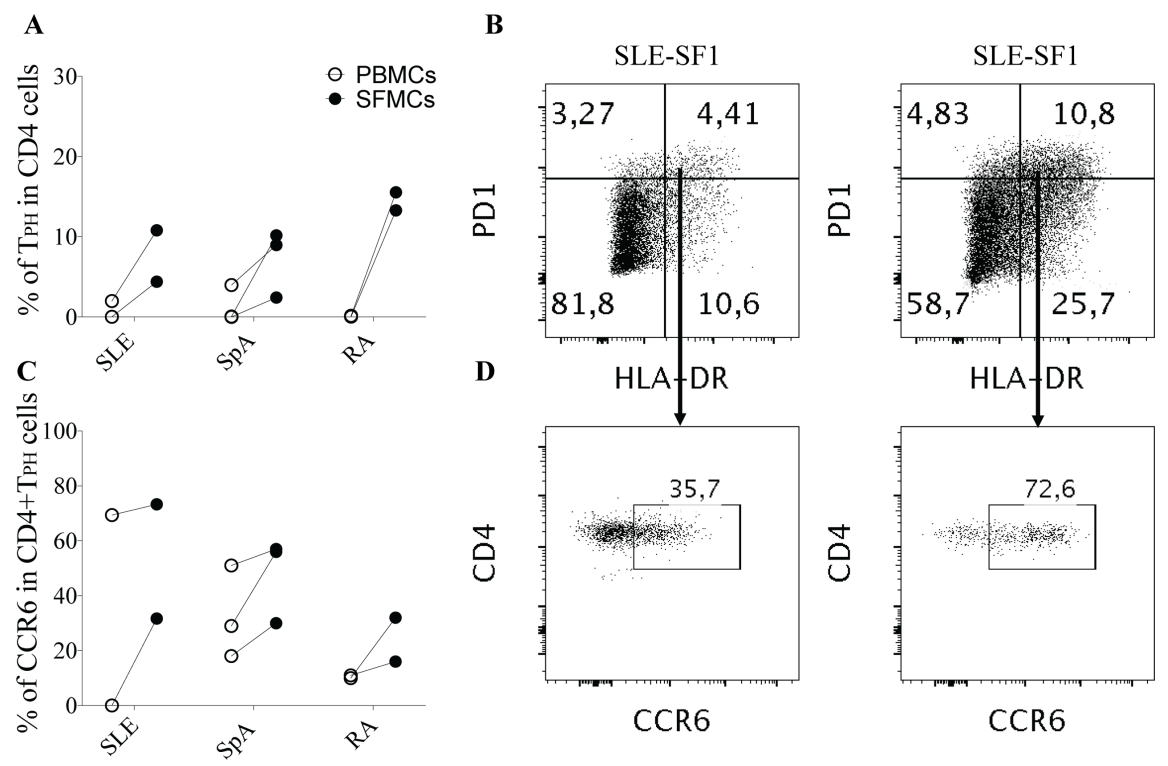

\section{Figure 3:}

T peripheral helper (TPH) cells in SF of SLE patients (A) Frequency of $\mathrm{T}_{\mathrm{PH}}$ cells in SF and serum samples in SLE $(n=2), \operatorname{SpA}(n=3)$ and age and sex matched RA $(n=2)$ patients. (B) Gating plots of the $\mathrm{SF}$ samples of the two SLE patients. $\mathrm{T}_{\mathrm{PH}}$ cells are defined as CD4+ PD- ${ }^{\text {high }} \mathrm{HLA}-\mathrm{DR}{ }^{\text {high }}$ cells gated by quadrant gating. (C) Frequency of CCR6+ expression in $\mathrm{T}_{\mathrm{PH}}$ cells in the three different diseases (D) SLE-SF plots showing CCR6 gates after gating for CD4+ PD- high $^{\text {HLA-DR }}{ }^{\text {high }}$ cells.

A
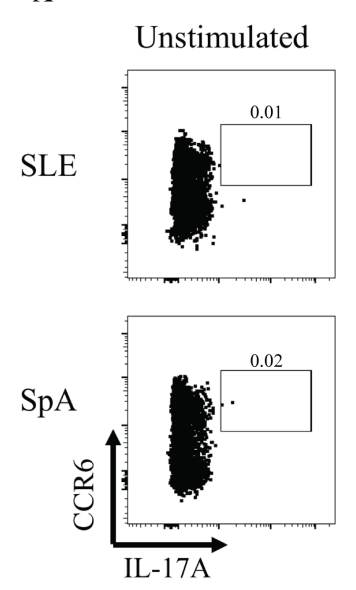

B
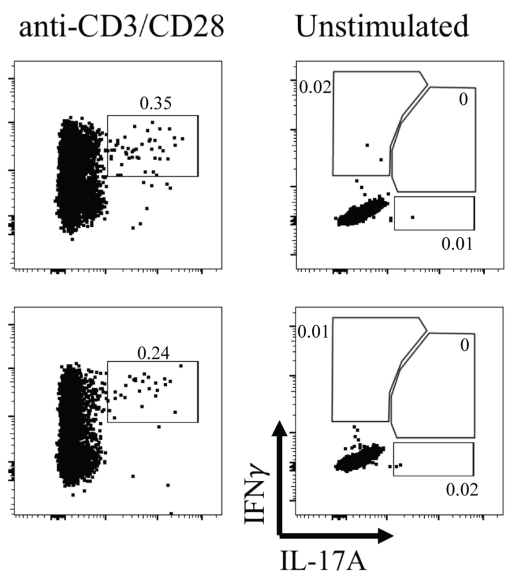

anti-CD3/CD28
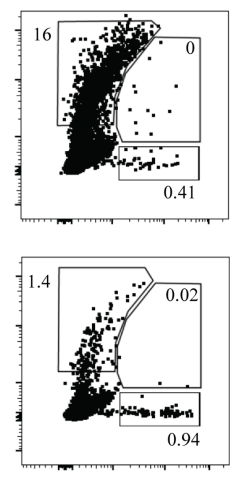

Figure 4: 
T-cell stimulation of SFMC from SLE and SpA Intracellular flow cytometry dot plots of (A) CCR6 vs IL-17A and (B) IFN? vs IL-17A in stimulated SFMC ( $n=1$ SLE and $n=1$ SpA) The cells were stimulated over night with anti-CD3/CD28 beads.

\section{Acknowledgements}

We would like to thank Susana Hernandez Machado, Gloria Rostvall and Julia Norkko, for helping with sample collection and management and Vijay Joshua for helping with the CBA software. This study was conceived, designed and coordinated by NS, FF, KC, IG; VM. FF and ST contributed to data collection and clinical assessments. Experimental work was performed by NS in supervision of KC and VM. Rheumatoid Factor data was obtained by JR. The data analysis and interpretation were conducted by NS, FF, KC, VM, IG and the writing was drafted by FF and NS. This work was supported by The King Gustaf V 80th Birthday Fund, Stockholm County Council (ALF and for ST: combined clinical residency and PhD training program), The Swedish Rheumatism Association, Ingegerd Johansson's Foundation, and The Swedish research council.

\section{Disclosures}

The authors have no conflict of interest to disclose.

\section{Ethical statement:}

Our study complies with the Declaration of Helsinki and has been approved by locally appointed ethical committee. 